\title{
Creencias de las profesoras de la Fundación Íntegramente. Un primer acercamiento al contexto sociocultural de la enseñanza de castellano a los inmigrantes de Santa Coloma de Gramenet
}

Hermes Iván Díaz Ceniceros, Universitat de Barcelona, España

\section{Introducción}

La Fundación Integramenet es un centro patrocinado por la obra social la Caixa que se dedica principalmente a la inserción laboral de inmigrantes en el barrio del Raval de Santa Coloma de Gramenet. Sin embargo, esta fundación ha observado que para lograr lo anterior es necesario que estos inmigrantes tengan conocimientos básicos de castellano, por este motivo se preocupa por la enseñanza de esta lengua a aquellos inmigrantes que no lo sepan hablar y lo necesiten para encontrar trabajo y adaptarse a la sociedad española. Por este motivo se crean las clases de castellano para inmigrantes con profesores voluntarios. En estas aulas los profesores voluntarios se enfrentan a la diversidad cultural y a distintos niveles educativos sin tener muchas veces una experiencia docente que pueda servirles para poder enseñar una L2 a un alumnado tan complejo. Por este motivo nos preocupamos por conocer los sistemas de creencias de estos profesores, con el propósito de acercarnos a este tipo de aulas y de ver cómo estos se adaptan o no al contexto multicultural.

\section{Base Teórica}

Las investigaciones sobre las creencias no es algo nuevo para la antropología y la psicología; sin embargo, como van Lier señala en el prefacio del libro de Kalaja y Barcelos, Beliefs about SLA. New research approaches (2006), en el campo de la educación estas investigaciones comenzaron a aparecer en la década de los ochenta. En este tiempo, se han formulado tres maneras de aproximarse al estudio de las creencias: la manera normativa, la metacognitiva y la contextual. En este trabajo nuestra aproximación será desde el enfoque contextual debido a que al interesarnos por aspectos culturales como la identidad, creemos que la enseñanza de una L2 es también la transmisión de una cultura. Para definir lo que entenderemos por creencias nos apoyaremos en la diferencia que Pajares (1992) hace entre sistemas de creencias y 
sistemas de conocimientos con el propósito de ver las características de las primeras. Posteriormente veremos cómo Ferreira Barcelos (2006) propone que las creencias toman forma en el contexto social donde se da el fenómeno de enseñanza-aprendizaje.

\section{¿Qué son las creencias?}

Pajares (1992) diferencia entre sistemas de creencias y sistemas de conocimientos observando, al igual que Nespor (1987) y Nisbet y Ross (1980), que los conocimientos son los pensamientos de una estructura cognitiva sistemáticamente organizados y externados, mientras que las creencias son un sistema de afecciones evaluadas, valoradas y externadas con algún significado cognitivo. Es decir los conocimientos se manifiestan en declaraciones sobre la información que se sabe de algún tema o de la realidad, mientras que las creencias son interpretaciones que manifiestan cierto grado de afección después de una evaluación y una valoración de algún tema o de la realidad. Pero la mayor diferencia entre los dos sistemas está en que el de conocimientos necesita la aprobación de un grupo y por tanto es cambiante; mientras que las creencias son inflexibles, menos dinámicas y más determinantes en cómo el individuo organiza y define sus actos.

Knowledge systems are open to evaluation and critical examination; beliefs are not. Nespor added that belief systems are also unbounded in that their relevance to reality defies logic, whereas knowledge systems are better defined and receptive to reason. And yet, for all their idiosyncrasies, he concluded that beliefs are far more influential than knowledge in determining how individuals organize and define tasks and problems and are stronger predicators of behavior. (Pajares 1992: 311)

Con esta observación podemos ver como el sistema de creencias se distingue del sistema de conocimientos en dos puntos: su naturaleza cuestionable y su relación con la realidad. Por nuestra parte nos apoyaremos en el siguiente cuadro para distinguir los dos sistemas en cuatro puntos: cuestionabilidad, flexibilidad, variabilidad y limites.

Cuadro 1. Características de los sistemas de creencias y de conocimientos

\begin{tabular}{|l|l|}
\hline Sistema de creencias & Sistema de conocimientos \\
\hline$\bullet$ Cuestionable por naturaleza & $\bullet$ Incuestionable \\
$\bullet$ Inflexible & $\bullet$ Dinámico \\
$\bullet$ Invariable & $\bullet$ Abierto a la crítica y evaluación \\
$\bullet$ Ilimitado & $\bullet$ Limitado \\
\hline
\end{tabular}


En el primer punto, la cuestionabilidad de las creencias o de los conocimientos se da en la relación de estos con la realidad. Los conocimientos son incuestionables debido a que se basan en datos evidentes de la realidad, mientras que las creencias son interpretaciones personales de esta y por tanto cuestionables por su subjetividad. La flexibilidad por su parte es la capacidad de cómo el individuo puede cambiar sus creencias o sus conocimientos de acuerdo a su relación con la realidad, en este sentido las creencias se manifiestan como inflexibles mientras que los conocimientos por su parte pueden cambiar por su carácter dinámico. En un caso similar, la variabilidad alude a la capacidad de un individuo para exponer a la crítica o a la evaluación tanto al sistema de creencias como al de conocimientos, en este sentido las creencias se exponen pocas veces a la critica por el mismo individuo que las posee, en cambio los conocimientos si son criticados y evaluados por el propio individuo con mayor frecuencia. Por último, los límites de los sistemas de creencias o de conocimientos se verán en lo razonables que estos llegan a ser, en este sentido lo ilimitado del sistema de creencias implica que este puede desafiar la lógica, mientras que por lo contrario, el sistema de conocimientos al ser limitado está obligado a limitarse a lo razonable.

Pajares (1992) propone un sistema de análisis de los sistemas de creencias que contempla tres aspectos: las creencias se diferencian en intensidad y poder; varían entre centrales y periféricas y mientras más central sea una creencia es más resistente al cambio. En esta propuesta la centralidad en las creencias es la capacidad de conectividad que estas tienen. En este sentido mientras mejor conectada o comunicada este la creencia, tendrá más importancia y por lo tanto será más intensa y poderosa debido a su centralidad en el sistema.

Ferreira Barcelos (2006) ve las creencias desde otras disciplinas y nos indica que este concepto no es sólo cognitivo, sino que también es un constructo social que nace de experiencias y problemas. Tomaremos de Ferreira Barcelos (Op. Cit.) tres propuestas para ver como las creencias emergen en un entorno social: la teoría de Deweyan sobre el conflicto y la influencia, las creencias en el diálogo, y la construcción social de las creencias en el aula. La teoría de Deweyan propone que la enseñanza y el aprendizaje son procesos continuos de reconstrucción de experiencias, en los cuales estamos constantemente interpretando la realidad y constantemente usamos nuestras creencias para darle sentido a los eventos y al ambiente que nos rodea. Por este motivo, desde esta 
teoría se entenderá por creencia toda forma de pensamiento que cubra las preocupaciones de aquello que no tenemos como conocimiento seguro, pero que nos parece suficientemente confiable como para actuar aceptándolo como cierto. Esto que se acepta, según Deweyan, se hace desde la interacción con las personas que nos rodean y por tanto con la creación de una identidad. Lo anterior nos permite entender que las creencias de los profesores son el producto de una constante interpretación de la realidad del aula. Esta interpretación no la hace solamente el profesor, sino que al compartir con los alumnos la misma realidad del aula, esta es interpretada desde distintos ángulos, ocasionando que las creencias de los profesores estén interrelacionadas con las de sus alumnos.

La segunda propuesta de Ferreira Barcelos (2006) tiene como punto de partida la idea de Dufva (2006) de analizar las creencias como producto del dialogismo. La propuesta de Dufva retoma el termino de "voice” de Bakhtin (1977) el que usa para nombrar todos los elementos alrededor del lenguaje en una conversación, dentro de los cuales están los significados, las opiniones, las actitudes, las reflexiones de la personalidad y por lo tanto las visiones del mundo. Analizar las creencias como el resultado de un proceso interacciones implica sostener que estas no son estáticas, que están determinadas por el contexto en el cual se producen y son polifónicas. En este sentido las creencias sólo aparecen cuando son articuladas de manera verbal o a través de signos, haciendo del lenguaje el vehículo mediador de las creencias. En este sentido el lenguaje transporta a las creencias de lo individual al discurso de otros o de la colectividad.

La última propuesta nos lleva al salón de clases y propone tres maneras de diferenciar los roles de las creencias en el aula: A) Las creencias no son discretas, están interconectadas y estructuradas. B) Las creencias no son identidades estables e individuales, están situadas en un contexto social formado a través de específicas instancias de interacción social. C) Las creencias no pueden ser separadas o son separables de otros aspectos del aprendizaje o del proceso cognitivo. Desde esta perspectiva Woods (2006) propone relacionar a las creencias con los procesos cognitivos del aprendizaje de lenguas y la interacción que se da entre los estudiantes y quienes cumplen la función del profesor. Desde esta perspectiva se ve al curso de L2 como un evento de habla debido a que se le entiende como un constructo social, 
conceptualizado como una unidad. De esta manera el modelo BAK (beliefs, assumptions and knowledge) propuesto por Peter Woods (1995), nos vuelve a remitir al hecho de que nada ocurre fuera de su contexto, así como que el planteamiento y el proceso de interpretación siempre debe ser contextualizado.

\section{La ecología en el aprendizaje de la lengua, la metodología cualitativa y el enfoque etnográfico}

Según Pulido (1995) uno de los puntos de partida de la investigación etnográfica en la educación es reconocer a la escuela como un agente de transmisión de la cultura. En nuestro caso no trabajaremos con una escuela estrictamente, pero sí en un entorno educativo donde se enseña una segunda lengua, enmarcado por un entorno social y cultural definido. Cuando consideramos a la escuela o a un centro de educación como un agente de transmisión de cultura, tenemos que reconocer cómo esta usa el lenguaje en todas las actividades, tanto en las académicas como en las extra académicas, que pueden ser desde la evaluación y las actividades dentro del aula. Tomando lo anterior en cuenta, van Lier (2004) propone que el lenguaje en la escuela debe ser visto como parte de un sistema de mensajes que está relacionado con todo el sistema sensorial, las memorias e historias que sirven para construir una identidad y un sistema de creencias. Desde este enfoque van Lier le propone dar a las ciencias de la educación un enfoque ecológico en el cual se ve al aula como un nicho ecológico.

A través de este enfoque las herramientas etnográficas empleadas en las ciencias de la educación dejan de ser sólo una metodología para obtener datos cualitativos y analizarlos. La etnografía educativa se debe centrar en descubrir lo que sucede cotidianamente en la escuela. En este sentido el etnógrafo no debe quedarse en la dimensión descriptiva exclusivamente, ahora se le permite sugerir alternativas, teorías y prácticas que lleven a una mejor intervención pedagógica. En nuestro caso no trabajaremos como evaluadores etnográficos y nos limitaremos a la descripción, con el propósito de exponer los datos analizados de manera que después puedan ser reutilizados en un trabajo más amplio, en el cual se puedan hacer propuestas prácticas que mejoren el trabajo de los profesores voluntarios. 


\section{Herramientas de investigación}

Las herramientas de investigación etnográfica en las ciencias de la educación pueden ser utilizadas tanto por el profesor como por un investigador ajeno a la escuela. Independientemente de quien las utilice estas permiten desarrollar un enfoque reflexivo y crítico de la enseñanza. El fundamento metodológico de estas herramientas tiene su origen en la antropología lingüística, ciencia que se interesa en la comunicación e interacción de los individuos a través del lenguaje en la cultura que construyen. Para Tusón (1995) esta manera de practicar la etnografía se basa en qué hacen social y culturalmente las personas por medio de la comunicación lingüística, reconociendo al discurso como una práctica social con características complejas. En este sentido se reconoce en el discurso la diversidad de modos de organización y por el otro la heterogeneidad con la cual se regula con una serie de normas, principios o máximas de carácter textual y sociocultural.

Para obtener los datos utilizaremos como herramienta de recolección la entrevista etnográfica. Al igual que Hammersley y Atkinson (1983) consideramos a ésta como una de tipo semi-abierta en la cual las preguntas no están totalmente definidas de antemano pero sí giran en torno a una serie de temas definidos con anterioridad. Una vez recolectados los datos usaremos el sistema de análisis de Hymes (1971) SPEAKING (situation, participants, ends, act sequences, key, instrumentalities, norms and genre) debido a que este sistema entiende como competencia comunicativa todo lo que los hablantes necesitan saber para comunicarse de manera eficaz en contextos culturalmente significativos. Este sistema pone énfasis en distinguir entre lo que un hablante sabe y la manera cómo se comporta en situaciones particulares. Para hacer esta distinción se entiende que el acto comunicativo es una opción tomada de un conjunto entre las cuales la persona debe escoger cuándo y cómo hablar guiándose por determinados hábitos, normas, principios de carácter sociocultural propios del grupo al cual pertenece. Retomando lo anterior, Tusón (1995) propone ver la escuela y el aula como un escenario comunicativo en el sentido de que se da uso de códigos comunicativos verbales y no verbales. De esta manera se presenta el aula como un microcosmos donde se concentran las relaciones típicas de la sociedad de la cual se forma parte y donde hay relaciones jerárquicas y entre iguales, de poder y de solidaridad; así como íntimas y distantes. 


\section{Planteamiento de las preguntas de investigación}

Una vez ya definidas las características de las creencias, haciendo notar su inflexibilidad y su invariabilidad dependiendo de la centralidad de estas, así como también su construcción social en el aula, las preguntas de investigación se enfocan en indagar cuáles son las creencias de los profesores voluntarios y la forma en la cual estas afectan las tomas de decisiones del profesor. Los temas en los cuales nos centramos para ver cómo las creencias de los profesores voluntarios afectan en la toma de sus decisiones son cuatro: a) la relación entre la profesión y las creencias; b) cómo el aprendizaje de una segunda lengua sirve de experiencia previa; c) la valorización de la multiculturalidad; d) cómo se identifican con los problemas de los estudiantes por su condición de inmigrantes.

En torno al tema de la relación entre la profesión y las creencias de los profesores nos interesa conocer cómo la profesión influye en las creencias con las que los profesores voluntarios llegan al aula. Tomando en cuenta que los profesores voluntarios tienen como característica trabajar o haber trabajado en otras partes, creemos que esto los hace manejar discursos distintos y por lo tanto tener puntos de vista diferentes sobre la experiencia como voluntarios. Con todo esto creemos que la profesión es un aspecto importante en el profesor voluntario que determina muchas de las creencias, así como de la manera en la cual se enfrentan a los problemas que aparecen.

Teniendo en cuenta la realidad lingüística de Cataluña no fue posible dejar a un lado plantearnos cómo se usa la experiencia del aprendizaje de una L2 para preparar clases. Esta característica lingüística de los profesores nos lleva a interesarnos en cómo la experiencia de aprender una L2 afecta en las creencias de estos.

En cuanto al tema de la multiculturalidad nos interesa conocer las ideas adelantadas que los profesores tienen de los grupos con los que trabajan. Por lo tanto queremos reconocer cuáles de los siguientes aspectos: edad, profesión, sexo y nacionalidad; son los que más influyen en la valoración de la multiculturalidad y como se usa tanto para enfrentar problemas como para prepara las clases. Así pues, también 
buscaremos conocer cómo y en qué medida se da la identificación del profesor con sus estudiantes.

\section{Definición del corpus de datos}

Las entrevistas que usaremos son cinco en total, las cuales se les hicieron a cuatro informantes. La primera entrevista, hecha a la informante entrevistada dos veces, fue en diciembre del 2008 antes de que pensáramos en este trabajo. Por este motivo la entrevista no fue diseñada para obtener los datos de todos los temas que nos interesan en este trabajo, sin embargo aparecen datos importantes que no pudimos dejar de tomar en cuenta. En este sentido lo que decidimos fue llevar a cabo una segunda entrevista a la misma informante con el propósito de recoger los datos que no obtuvimos de la primera entrevista. Por otra parte, las cuatro entrevistas restantes fueron realizadas en mayo del 2009.

Las informantes son cuatro mujeres profesoras voluntarias en la Fundación Integramenet. Las edades entre ellas son distintas, al igual que sus profesiones, además de que no todas comparten la misma nacionalidad. Para proteger su identidad todas tendrán un pseudónimo.

\section{Tere}

Fue la primera en ser entrevistada en diciembre del 2008, es la más joven de las cuatro con 30 años. Nació en México pero lleva viviendo en Barcelona más de nueve años. Su profesión es la de administración de empresas y trabaja en la Fundación Integramenet en las oficinas administrativas. Lleva alrededor de cinco años como voluntaria y la misma cantidad trabajando para la fundación. Tiene como primera lengua el castellano, aprendió en México el inglés y en Barcelona el catalán.

\section{Zafo}

Cuando se hicieron las entrevistas a todas las profesoras voluntarias Zafo fue la primera en acceder. Ella es una mujer de 47 años. Su profesión es la de profesora de lengua. Lleva cinco años de voluntaria y ha recibido clases de francés, ingles, italiano y alemán. Su lengua materna es el catalán y aprendió el castellano como lengua oficial.

\section{Helen}


Es una monja que se encuentra en la tercera edad. Es la voluntaria que más tiempo lleva en la fundación con ocho años. En cuanto a la lengua ella se define como hablante del castellano y ha aprendido el catalán por vivir en Barcelona, así también ha recibido clases de ingles, francés y árabe.

\section{Sofía}

Al igual que Helena también es una monja de la tercera edad, sin embargo no lleva tanto tiempo como voluntaria y hasta el momento de la entrevista solo llevaba cuatro años. En cuanto a la lengua, Sofía reconoce que en su casa aprendió el castellano, sin embargo también se siente como hablante nativa del catalán. A lo anterior se le une unas clases de francés que llevó de pequeña aunque ahora piensa que no lo domina.

El único requisito que quisimos que cumplieran nuestras informantes fue la antigüedad como profesores voluntarios en la fundación. Las variantes de la edad, el sexo y la profesión no las tomamos en cuenta en la selección como características que los informantes deban de tener en común porque creemos que dejar esta variedad de características iba a permitir una riqueza de datos. Aun con la búsqueda de variantes, encontramos que todas las profesoras con mayor antigüedad en la fundación son mujeres. Con respecto a la profesión Helena y Sofía son monjas, mientras que Tere y Zafo se diferencian también en este terreno, la primera como administradora de empresas y la segunda como profesional de la docencia. Por último con respecto a la lengua, como supusimos, cada una de las informantes ha pasado por el proceso de adquirir una segunda lengua, ya sea por gusto o por vivir en una ciudad donde la situación lingüística exige a sus habitantes ser bilingües.

Tres de las cuatro entrevistas fueron en el mismo lugar, el salón donde se dan los cursos de español y después de que el profesor había terminado su clase. Las dos entrevistas a Tere fueron hechas en las oficinas de la fundación, donde ella trabaja. El motivo por el cual a Tere se la entrevistó en su lugar de trabajo fue porque ella trabaja en la misma fundación que ofrece los cursos y en lugar de ir detrás de ella al final de su clase, preferimos entrevistarla en su oficina por comodidad. 


\section{Análisis de los datos}

En las entrevistas con cada una de las profesoras voluntarias reconocimos posiciones distintas en cuanto a los temas que nos planteamos al principio. En el caso de Tere encontramos que su discurso lo configura como el de una persona mediadora usando expresiones como: “intentar hacer como | un pequeño bálsamo entre ellos”. Por otra parte observamos que su profesión influye en gran medida en su discurso como voluntaria, llevándola a ver a su trabajo en la fundación y a su labor de voluntaria como “dos puntas de lanza” para atacar la problemática que viven los inmigrantes de la comunidad. Sin embargo, a pesar de lo anterior, Tere constantemente intenta marcar una distancia entre su experiencia migratoria y la que viven sus alumnos.

Fragmento 1:

11TER: no | ((sonríe)) porque yo nunca fui a clases de catalán | yo lo aprendí viendo la televisión y en las clases de la universidad | pero claro clases muy distintas de contabilidad:: | administración:: | economía:: | y lo aprendí así el catalán y leyendo también en catalán | viendo la televisión | escuchando música | yo soy | aprendí catalán de la calle no | no | nunca fui a clases |nunca fui a clases |

En esta respuesta se observa como la informante cree que su experiencia aprendiendo una L2 no le es útil para enseñar debido a que cree que fue muy diferente a la que viven sus alumnos por las diferencias entre su condición migratoria y la de ellos. En este sentido Tere marca las diferencias que existen entre los inmigrantes que llegan en busca de trabajo dese países de magrebís, África y Asía y aquellos, que como ella, llegan como estudiantes desde países latinoamericanos.

En el caso de Zafo encontramos un discurso muy marcado por su profesión como docente y por tanto es quien reflexión más profundamente sobre la experiencia de haber aprendido una L2 y como ésta puede usarse para preparar clases.

Fragmento 2:

9ZAF: / no I haber / yo a:: I incluso ahora / no solo al principio / a:: ((aplaude)) si me pongo a mi misma / cuando yo he estudiado otras lenguas no / y entonces I he aplicado / más o menos I los mismos métodos \actividades \ y procesos / con los que yo he aprendido otras lenguas $\backslash$ e::

10ENT: a vale

10ZAF: lo que pasa es que esto / me sirve:: para:: $\backslash /$ alumnos de lenguas europeas $\backslash$ o más o menos próximas

11ENT: si 
11ZAF: pero claro no me sirve para / chinos o gente que no sabe ingles por ejemplo \

Estas reflexiones en torno a la adquisición de una L2 la llevan a no diferenciar a sus alumnos por países, religiones u orígenes étnicos, sino por su nivel de comprensión. Por último Zafo encuentra inquietante las distancias entre las lenguas de sus alumnos y las lenguas con un alfabeto europeo, cree que esta distancia dificulta mucho el proceso de aprendizaje.

Tanto en el discurso de Helena como en el de Sofía encontramos influencia de la vocación religiosa, sin embargo creemos que esta se manifiesta de forma distinta en cada una de ellas. En el caso de Helena su vocación religiosa se deja notar en la manera en la cual maneja un discurso compasivo que apela a la concepción cristiana de amar al prójimo como a uno mismo y al ideal de austeridad de algunas congregaciones religiosas que buscan vivir con los que menos tienen o sufrir con el desamparado tratando de emular la vida de Cristo. "haber m:: de empatía / si I de I de sufrir con ellos / si I d..de situaciones de falta de trabajo que están viviendo y que lo están sufriendo también” (2009). En este se puede ver como constantemente intenta ponerse en el lugar de sus alumnos. Esto también la lleva encontrarle utilidad a su experiencia aprendiendo un L2, pero no de una manera reflexiva como en el caso de Zafo, sino de una manera emocional. "pues también me I me ayuda I porque yo misma conozco las dificultades que yo he tenido para aprender el otro idioma y me ayuda en ese sentido” (2009). Por su parte, en el discurso de Sofía encontramos la influencia de la vocación religiosa en un discurso que se preocupa por la educación cívica del alumnado en un tono doctrinario.

Fragmento 3:

claro e:: e:: bueno pues esto esta:: lo que a veces yo interrumpo el I el I lo:: que es e:: I haber lo propio de la lengua no/ I el conocimiento I y entonces pues te pones hacer discurso I $\backslash$ haber un discurso I / que yo digo mira como venís a aprender lengua interesa que aprendáis vocabulario I que me preguntéis lo que no entendéis I pero hoy me vais a escuchar I y entonces aprovecho e:: diciendo II aspectos I que tienen que corregir I que tiene que mejorar I como tiene que:: que darse cuenta también I que los que somos de aquí I no todo lo hacemos bien II que han de tener I han de ser lucidos I me sale la palabra lucido con ellos voy buscando otro vocabulario e:: I para que me inters... I para que me entiendan I ser lucidos para distinguir / que es lo bueno que yo me encuentro aquí en esta tierra que me acoge / I para ello I también distinta de la mía I pero que me dé cuenta que es un valor que es bueno y que yo lo / quiero I $\backslash$ pero hay cosas que no I y yo les digo I no las aprendáis II costumbres que no I pero eso no es cultura 
I estos son e e haber e:: por ejemplo I yo les digo e:: coges el metro I vas a coger el metro I corren se ponen allí I ponen una pierna encima del otro asiento I y vosotros veis que hay muchos de aquí que lo / hacen I luego tenéis que tener un criterio de decir I hombre esto está mal II pero a veces vosotros venís y copiáis I es decir II curiosamente las cosas que no están bien I enseguida les cala II y las llegan a imitar I todo esto I pues yo un poco I aprenden vocabulario cosas que no I y entonces vuelvo a repetir

Sin embargo este tono doctrinario que se manifiesta en la preocupación por el comportamiento moral y cívico del alumnado, en la cual es evidente una necesidad por indicar lo que se considera bueno y malo de acuerdo con sus creencias, se contradice con el constante intento por parte de Sofía de aparentar una apertura total a la multiculturalidad. Al final, al igual que Tere, tampoco encontró similitudes entre su experiencia aprendiendo una L2 y la de sus alumnos.

\section{Resultados}

Observamos que la relación entre la profesión y las creencias afectan en como el profesor voluntario se identifica con los estudiantes, la manera en la cual valoriza la multiculturalidad e incluso el uso de la experiencia aprendiendo una L2 para preparar clases. Por este motivo concluimos que la profesión es el factor de mayor influencia en el sistema de creencias de estas profesoras voluntarias. En este sentido podemos ver que el sistema de creencias de los profesores voluntarios depende de su relación con las creencias que se generan en su vida laboral.

Debido a que este trabajo tenía el propósito de tener un primer acercamiento a la Fundación Integramanet con el fin de conocer al tipo de profesores voluntarios que trabajan allí y al mismo tiempo mantener un primer contacto con las aulas multiculturales, nos limitamos a una descripción etnográfica. Sin embargo, creemos que los datos presentados aquí dan pie para una investigación más amplia en la cual se contemple también el sistema de creencias de los alumnos. En este sentido pensamos que los sistemas de creencias de los profesores vistos en este trabajo deben ponerse frente a los sistemas de creencias de los estudiantes de este tipo de aulas con el propósito de verlos en su justa dimensión. Para ello es necesario seguir haciendo trabajo de campo etnográfico en estas aulas, hacer observaciones de clases, usar las herramientas de los diarios de clases y las historias de vida lingüística de los estudiantes 
para triangular datos y tener una imagen más próxima a la realidad que se vive en estas aulas. Por este motivo, a partir de los datos presentados en este trabajo, queremos seguir investigando para ver cómo los alumnos perciben el sistema de creencias de los profesores. Creemos que las creencias religiosas y las diferencias entre las expectativas de cómo se debe aprender una L2 entre los profesores y los alumnos es un factor importante para el aprovechamiento de estas clases por parte del alumnado. De esta manera, sostenemos que a medida que conozcamos mejor el contexto de estas aulas, a partir de la investigación etnográfica, podremos crear elementos de apoyo que realmente le sirvan a los profesores voluntarios. Quienes a pesar de su entusiasmo en ayudar a un alumnado tan complejo, necesitan de recursos diseñados para ellos y su contexto.

\section{Referencias bibliográficas}

Dufva, H. (2006) Beliefs in dialogue: A Bakhtinian view. In: Ferreiro Barcelos, A. M. (ed.) Researching beliefs about SLA: A critical review, pp.131-152. Dordrecht: Kluwer Academic Publishers.

Bakhtin, M. (1977) Le marxisme et la philosophie du langage. París: Les Éditions de minuit. [1a ed., 1929] Francia.

Ferreira Barcelos, A. M. (2006) Teachers' and students' beliefs within a Deweyan framework: Conflict and influence. In: Ferreiro Barcelos, A. M. (ed.)

Researching beliefs about SLA: A critical review, pp.171-200. Dordrecht: Kluwer Academic Publishers.

Hammersley M. y Atkinson P. (1983) Ethnography: Principles in practice. London \& New York:Tavistock Publications.

Hymes,D. (1971) On communicative competence. Filadelfia, USA: University of Pennsylvania Press.

Kalaja, P. y Ferrera Barcelos, A.M. (2006) Beliefs about SLA. New research approaches. In: Ferreiro Barcelos, A. M. (ed.) Researching beliefs about SLA: A critical review, pp. 231-240. Dordrecht: Kluwer Academic Publishers.

Nespor, J. (1987) The role of beliefs in the practice teaching. Journal Curriculum Studies, 19: 317-328.

Nisbet, R.E. y Ross, L. (1980) Human inference: Strategies and shortcoming of social judgment. Englewood Cliffs (NJ): Prentice-Hall.

Pajares, M. F. (1992) Teachers' beliefs and educational research: cleaning up a messy Construct. Review of Educational Research, 62 (3): 307-332.

Pulido Moyano, R.A. (1995) Etnografia i investigació educativa: concepcions esbiaixades, relacions malenteses, Temps d'Educació: 11-31.

Tusón Valls, A. (1995) L’etnografia de la comunicació i la investigació educativa: l'aula com microcosmos. Temps d'Educació, 14: 149-161.

Van Lier, L. (2004) The ecology and semiotics of language learning. Dordrecht: Kluwer Academic Publishers. 
Woods, D. (2006) The social construction of beliefs in the language classroom. In: Ferreiro Barcelos, A. M. (ed.) Researching beliefs about SLA: A critical review, pp. 201-230. Dordrecht: Kluwer Academic Publishers.

Woods, P. (1995) Aprofundir e la vida de les coses: l’etnografia a la investigación educativa. Temps d'Educació, 14: 107-132.

Referencia del autor:

Hermes Iván Díaz Ceniceros es estudiante de nacionalidad mexicana en el programe de doctorado en didáctica de la lengua y la literatura en la Universidad de Barcelona y becario de la AECID gracias al programa de becas MAEC-AECID. En septiembre del 2009 termina el máster oficial en Investigación en didáctica de la lengua y la literatura con una memoria dirigida por la Dra. Carolina Forns y de la cual sale este articulo.

Email: bubagump001@hotmail.com 